\title{
Enhancing Case Detection of Selected Inherited Disorders through Expanded Newborn Screening in the Philippines
}

\author{
Carmencita D. Padilla*1,2 \\ *Tomas B. Aguirre Professorial Chair in Pediatrics \\ ${ }^{1}$ Department of Pediatrics, College of Medicine and Philippine General Hospital, University of the Philippines Manila \\ ${ }^{2}$ Newborn Screening Reference Center, National Institutes of Health, University of the Philippines Manila
}

\begin{abstract}
Background. Newborn screening in the Philippines currently includes screening for 6 disorders - congenital hypothyroidism $(\mathrm{CH})$, congenital adrenal hyperplasia (CAH), phenylketonuria (PKU), glucose-6-phosphate dehydrogenase (G6PD) deficiency, galactosemia (GAL) and maple syrup urine disease (MSUD). With improved newborn screening technologies, the potential of screening more than 70 disorders has become a possibility. Worldwide, there has been a move towards increasing the number of disorders included in newborn screening panels. The California Newborn Screening Program (CNSP) screens for over 70 disorders and its database includes a large number of Filipino newborns.
\end{abstract}

Objectives. To describe the profile of Filipino newborns screened through the CNSP and to extrapolate these data to the Philippine newborn population in order to assess the potential value of expanding the Philippine newborn screening program.

Methods. The newborn screening database of the CNSP was reviewed. Projections based on the California data were made relative to expanded newborn screening and related outcomes in the Philippines.

Results. From 2005 to 2011, a total of 3,460,839 newborns were screened in the CNSP which included 111,127 Filipinos. Among the Filipinos, there were 199 confirmed having one of the screened disorders categorized as follows: endocrinologic disorders (51); hemoglobinopathies (109); amino acid disorders (6); organic acid disorders (7); fatty acid disorders (10); and other disorders (16). Extrapolating these findings to the Philippine newborn population predicts the detection of significant additional cases of screened disorders including: 2180 hemoglobinopathies, 140 organic acid disorders, 200 fatty acid disorders, and 240 other disorders.

Presented at the 10th Newborn Screening Convention, Oct. 2, 2012, SMX Convention Center, Pasay City.

Corresponding author: Carmencita D. Padilla, MD

Newborn Screening Reference Center

National Institutes of Health

University of the Philippines Manila

625 Pedro Gil St, Ermita, Manila, Philippines

Telephone: +6325224396

Email: cdpadilla@upm.edu.ph
Conclusion. Data from the CNSP show serious disorders detected by newborn screening in Filipino babies that are not currently included in the limited newborn screening program in the Philippines. Expanding the panel of screened disorders to approximate that in the CNSP will result in significant additional case detections in the Philippines that will save lives and reduce unnecessary negative health outcomes through early detection and treatment.

Key Words: newborn screening, expanded newborn screening, Philippine newborn screening program

\section{Introduction}

Newborn screening (NBS) is a public health activity aimed at the early identification of infants who are affected by certain genetic/metabolic/infectious conditions. Early identification of these conditions is crucially important, since timely intervention leads to significant reduction of morbidity, mortality, and associated disabilities in affected newborns. ${ }^{1}$ Newborn screening for phenylketonuria (PKU), using a bacterial inhibition assay developed by Robert Guthrie in the early 1960s using blood absorbed onto a special filter paper, set the framework for NBS. ${ }^{2}$ Successful newborn screening for PKU and improved micro detection techniques for other conditions led to the gradual inclusion of other disorders in NBS programs over the years. Presently, both developed and developing countries include newborn screening as a standard of care covering a wide range of conditions - endocrinology, metabolic, hematologic, immunologic, infectious, and many others. ${ }^{3}$ This includes the national newborn screening program in the Philippines. The main goal of newborn screening is to detect and treat asymptomatic heritable disorders early in order to decrease related morbidity and mortality. ${ }^{4}$ Multiplex newborn screening assays for the hemoglobinopathies developed in the 1980s and metabolic disorders developed in the 1990s have led to significant additions to newborn screening panels in recent years. The extent to which these particular disorders affect Philippine newborns is not known; however, clinical case data in the Philippines and data on Filipino newborns in California provide incidence data useful in assessing probable incidences of the hemoglobinopathies (i.e. thalassemias) and other metabolic conditions (i.e. 
organic acid and fatty acid oxidation disorders) not currently part of the Philippine newborn screening panel.

\section{Newborn Screening in the Philippines}

Formalized NBS in the Philippines began in June 1996, and was integrated into the public health delivery system eight years later with enactment of Republic Act 9288, also known as the Newborn Screening Act of 2004. A National Comprehensive Newborn Screening System (NCNBSS) was established under RA 9288 to ensure that every baby born in the Philippines is offered the opportunity to undergo newborn screening and thus be spared from the adverse outcomes of a limited number of heritable conditions that can result in mental retardation and death if left undetected and untreated. ${ }^{4,5}$ By the end of 2011, newborn screening was being offered to $42 \%$ of the Philippine newborn population. The Department of Health (DOH) and other program implementers have been, and continue to be, aggressive in identifying strategies that have intensified newborn screening awareness in communities and increased screening coverage for home deliveries, which account for $60 \%$ of the newborn population. ${ }^{6}$

As of December 2012, six disorders are included in the NCNBSS. The incidences of the screened disorders are: congenital hypothyroidism $(\mathrm{CH})$ 1:3,004; congenital adrenal hyperplasia (CAH) 1:10,604; phenylketonuria (PKU) 1:388,367; galactosemia (GAL) 1:310,694 and glucose-6phosphate dehydrogenase (G6PD) deficiency 1:50. Maple syrup urine disease (MSUD) was added to the screening panel in the last quarter of 2012 and incidence data is not yet available. ${ }^{6}$

Samples are being collected from over 4,000 newborn screening facilities (NSFs) nationwide (including birthing centers, lying-in centers, rural health units, infirmaries, secondary/tertiary hospitals). NSFs offer NBS as part of $\mathrm{DOH}$ and Philippine Health Insurance Corporation (PHIC) licensing requirements for hospital accreditation. These facilities assist the newborn screening effort by educating parents about NBS during prenatal classes, collecting blood samples for NBS, sending samples to Newborn Screening Centers (NSCs), recalling patients found to be at increased risk through the screening process, and assisting in the referral of screen positive patients for proper management. Four Newborn Screening Centers (NSCs) currently provide laboratory and follow-up services for more than 4,000 NSFs. The NSCs are strategically located to provide newborn regional screening services - NSC-National Institutes of Health in Manila; NSC-Visayas in Iloilo City; NSCMindanao in Davao City; and NSC-Central Luzon in Angeles City. ${ }^{6}$

A summation of the major activities and accomplishments of the NCNSP across time are presented in Table 1 .
Table 1. Milestones of the Newborn Screening Program in the Philippines

\begin{tabular}{|c|c|}
\hline Year & Milestone \\
\hline 1996 & $\begin{array}{l}\text { Pilot screening initiated for } \mathrm{CH}, \mathrm{CAH}, \mathrm{GAL}, \mathrm{PKU}, \\
\text { homocystinuria (HCY). }\end{array}$ \\
\hline 1998 & Pilot screening initiated for G6PD deficiency. \\
\hline 1999 & $\begin{array}{l}\text { HCY discontinued (no cases detected) and replaced with G6PD } \\
\text { deficiency (shown to have high incidence in the pilot } \\
\text { study). }\end{array}$ \\
\hline 2000 & $\begin{array}{l}\text { Screening program evaluated for cost effectiveness and policy } \\
\text { changes recommended. }\end{array}$ \\
\hline 2004 & $\begin{array}{l}\text { Newborn Screening Act of } 2004 \text { enacted and the Implementing } \\
\text { Rules and Regulations completed. }\end{array}$ \\
\hline 2005 & NBS included in the licensing requirement of hospitals. \\
\hline 2006 & $\begin{array}{l}\text { NBS included in the Philippine Health Insurance Corporation } \\
\text { (PHIC) newborn package, covering } 90 \% \text { of the NBS fee. }\end{array}$ \\
\hline 2011 & PHIC newborn package expanded to cover $100 \%$ of the NBS fee. \\
\hline 2012 & $\begin{array}{l}\text { Maple Syrup Urine Disease (MSUD) added to Philippine NBS } \\
\text { panel. }\end{array}$ \\
\hline
\end{tabular}

\section{Expanded Newborn Screening}

In recent years, multiplex screening for hemoglobinopathies using electrophoresis, isoelectric focusing, and/or high performance liquid chromatography (HPLC), and for the amino acids, organic acids and acylcarnitines using tandem mass spectrometry (MS/MS) tremendously increased the number of detectable conditions in newborn screening. ${ }^{7,8,9,10}$ While hemoglobinopathy newborn screening has generally been limited to the US, expanded screening by MS/MS has been reported to vastly improve the outcomes from newborn screening in many countries including Australia, Europe and North America.11,12,13 Screening by MS/MS has approximately doubled the detection rate of metabolic disorders compared to that achieved by conventional methods. ${ }^{7}$ For some disorders (e.g. fatty acid oxidation disorders, MCAD deficiency) the detection rate is significantly higher by screening than by clinical diagnosis. ${ }^{11,14,15,16}$

Since neither hemoglobinopathy screening nor expanded metabolic screening are currently a part of the Philippine newborn screening program, questions exist as to whether their inclusion could and should be adopted. Is it worthwhile to expand the Philippine NBS Program? How many additional cases can be detected if this is done? While these questions could be the focus of a research project involving pilot screening, such a project would be costly and time consuming. A more efficient and equally effective method for answering these questions exists. Data from a large population of Filipino newborns in another wellestablished, high quality screening program, such as California, can be analyzed and extrapolated to the Philippine newborn population. The CNSP provides a source for this information. This report analyzes data on Filipino newborns screened through the CNSP and extrapolates case detection data to the Philippine newborn population (assuming full coverage of the 2 million newborns born each year). 


\section{Method}

Screening data for Filipino newborns born between 7 July 2005 and 6 July 2011 in California, USA included in the California Genetic Disease Screening Program database were obtained and analyzed. As a requirement of the CNSP, blood specimens were collected on all newborns by heelstick between 12 hours and 6 days after birth, with most specimens collected between 12 and 72 hours of age. Following collection, specimens were sent by the collecting hospitals to one of seven state-approved laboratories for testing. Screening for metabolic disorders was conducted using MS/MS to measure concentrations of acylcarnitines, organic acids and amino acids. Endocrine disorders [including congenital hypothyroidism $(\mathrm{CH})$ and congenital adrenal hyperplasia $(\mathrm{CAH})$ ] were screened by fluorescent immunoassays, similar to the procedure currently in place in the Philippines. Additional screening for $\mathrm{CAH}$ included second-tier testing by liquid chromatography coupled to MS/MS for androstenedione, cortisol, and 17hydroxyprogesterone. Hemoglobin disorders were screened using HPLC. Galactosemia and biotinidase deficiency (BD) were screened with fluorometric enzyme assays for galactose-1-phosphate uridyl transferase and biotinidase activity, respectively. CF was screened using a four-step approach (immunoreactive trypsinogen followed by a selected DNA mutation panel, followed by DNA sequencing, if the mutation panel identified two mutations, and a sweat test). Screening for CF and BD started on 16 June 2007 and thus, the data reported for these disorders represented only a 3-year interval. ${ }^{17}$

Information on race/ethnicity of the infants was reported by the mother, who was instructed to select all applicable choices from a selection of 18 distinct racial/ethnicity categories on the NBS test requisition form. ${ }^{17}$

All positive test results were reported to the California Genetic Disease Screening Program (GDSP) through a secure computer-based online Screening Information System. Newborns with initial screen-positive results were referred to a state-contracted specialty follow-up center for endocrine, metabolic, hemoglobin, or CF disorders. Center specialists determined whether referred children had a disorder through appropriate confirmatory testing. ${ }^{17}$

Data on the final diagnosis and ethnicity of the parents were collected from the database. The percentage of patients with the disorder was reported out of the total Filipino children screened and according to the ethnicity of the parents.

Extrapolations to the current Filipino newborn population in Philippines relied on birth statistics using an annual birth of 2 million (www.doh.gov.ph).

\section{Results}

From 7 July 2005 to 6 July 2011, there were 3,460,839 newborns screened in the CNSP including various races and ethnicities. Of these, 111,127 were Filipinos and 199 had confirmed diagnoses for screened disorders. Table 2 gives a breakdown of diagnoses among the Filipino patients confirmed with a disorder. Table 3 presents the ethnicity of the parents of the newborns. Table 4 presents the metabolic and hemoglobin disorders in Filipino newborns - the numbers picked up by the CNSP and extrapolated to annual number of cases assuming a 100\% coverage for the 2 million annual births.

Table 2. Diagnosis of confirmed cases in the Filipino newborn population in CNSP

\begin{tabular}{lccc}
\hline Type of Disorder & $\begin{array}{c}\text { Number } \\
\text { of Cases }\end{array}$ & $\begin{array}{c}\text { Percentage of } \\
\text { Cases among } \\
\text { Filipinos with } \\
\text { Confirmed } \\
\text { Diagnosis } \\
\mathbf{N = 1 9 9}\end{array}$ & $\begin{array}{c}\text { Percentage of } \\
\text { Cases among } \\
\text { Filipino } \\
\text { Population } \\
\text { Screened } \\
\mathbf{N = 1 1 1 ~ 1 2 7}\end{array}$ \\
\hline Endocrine Disorders & 51 & $26.6 \%$ & $0.05 \%$ \\
Hemoglobinopathies & 109 & $54.8 \%$ & $0.10 \%$ \\
Amino acid disorders & 6 & $3.0 \%$ & $0.01 \%$ \\
Organic acid disorders & 7 & $3.5 \%$ & $0.01 \%$ \\
Fatty acid disorders & 10 & $5.0 \%$ & $0.01 \%$ \\
Other disorders & 16 & $8.0 \%$ & $0.01 \%$ \\
Total & 199 & $\sim 100.0 \%$ & $\sim 0.18 \%$ \\
\hline
\end{tabular}

Table 3. Parental ethnicity of Filipino newborns with confirmed diagnosis in CNSPa

\begin{tabular}{lccr}
\hline \multicolumn{1}{c}{ Ethnicity } & $\begin{array}{c}\text { No. of } \\
\text { Filipinos }\end{array}$ & $\begin{array}{c}\text { No. of Filipino } \\
\text { patients with } \\
\text { Confirmed } \\
\text { Diagnosis }\end{array}$ & Percentage \\
\hline Filipinos only & 61,088 & 101 & $55.0 \%$ \\
Filipino + White & 18,546 & 26 & $17.0 \%$ \\
Filipino+ Hispanic & 8.507 & 13 & $7.6 \%$ \\
Filipino+ Hispanic + White & 3,849 & 6 & $3.4 \%$ \\
Other Filipinos & 19,127 & 53 & $17.0 \%$ \\
All Filipinos & 111,127 & 199 & $100.0 \%$ \\
\hline " personal communication with Lisa Feuchtbaum, DrPH, MPH, Genetic Disease \\
Screening Program, California Department of Public Health, Richmond, California, \\
USA, August 2012.
\end{tabular}

\section{Discussion}

Multiplex newborn screening tests for hemoglobinopathies in the $1980 \mathrm{~s}^{8}$ and metabolic conditions in the $1990 \mathrm{~s}^{18}$ have led to decreased morbidity and mortality in newborn screening programs adopting these techniques. Expanded newborn screening including hemoglobinopathies and metabolic conditions detected through multiplex assays has become newborn screening standard-of-care across the globe including: North America, ${ }^{13}$ Europe, ${ }^{12,19}$ Latin America, ${ }^{20}$ and Asia and the Pacific. ${ }^{21-26}$

Based on clinical case detection, there are a substantial number of Filipino patients with hemoglobinopathies. There are 400 patients registered in the Thalassemia Center of the Philippines in Quezon City and 30 patients registered with the Mindanao Thalassemia Group. Published data on 
Table 4. Metabolic and hemoglobin disorders in Filipino newborns: Data from the CNSP extrapolated to Philippine newborns

\begin{tabular}{|c|c|c|c|}
\hline Conditions & $\begin{array}{c}\text { Number of Cases } \\
\text { in CNSP }\end{array}$ & Prevalence & $\begin{array}{l}\text { Expected Annual Number of } \\
\text { Cases in the Philippines }\end{array}$ \\
\hline \multicolumn{4}{|c|}{ Hemoglobinopathies } \\
\hline$\alpha$-thalassemia major & 5 & $1: 22,225$ & 100 \\
\hline$\beta$-thalassemia major & 1 & $1: 111,127$ & 20 \\
\hline $\mathrm{Hb}$ E/Beta + thalassemia & 1 & $1: 111,127$ & 20 \\
\hline $\mathrm{Hb} \mathrm{H}$ disease & 93 & 1:1,195 & 1860 \\
\hline $\mathrm{Hb} \mathrm{H} /$ Constant Spring disease & 2 & $1: 55,564$ & 40 \\
\hline $\mathrm{Hb}$ Variant/Beta + thalassemia & 1 & $1: 111,127$ & 20 \\
\hline Homozygous EE & 3 & $1: 37,042$ & 60 \\
\hline Sickle C disease ( $\mathrm{Hb} \mathrm{S} / \mathrm{C}$ disease) & 2 & $1: 55,564$ & 40 \\
\hline Sickle cell anemia (Hb S/S disease) & 1 & $1: 111,127$ & 20 \\
\hline \multicolumn{4}{|c|}{ Amino Acid Disorders } \\
\hline Phenylketonuria (PKU) & 4 & $1: 27,782$ & 80 \\
\hline Variant hyperphenylalaninemia & 1 & $1: 111,127$ & 20 \\
\hline Maple syrup urine disease (MSUD) & 1 & $1: 111,127$ & 20 \\
\hline \multicolumn{4}{|c|}{ Organic Acid Disorders } \\
\hline Methylmalonic acidemia - MMA - (mut 0) & 3 & $1: 37,042$ & 60 \\
\hline Methylmalonic acidemia - MMA (mut -) & 2 & $1: 55,564$ & 40 \\
\hline$\beta$-ketothiolase deficiency (BKT) & 1 & $1: 111,127$ & 20 \\
\hline Isobutyryl-CoA dehydrogenase deficiency (IBDHD) & 1 & $1: 111,127$ & 20 \\
\hline \multicolumn{4}{|c|}{ Fatty Acid Oxidation Disorders } \\
\hline Medium chain acyl-CoA dehydrogenase deficiency (MCAD deficiency) & 2 & $1: 55,564$ & 40 \\
\hline Short chain acyl-CoA dehydrogenase deficiency (SCAD deficiency) & 3 & $1: 37,042$ & 60 \\
\hline Very long chain acyl-CoA dehydrogenase deficiency (VLCAD deficiency) & 3 & $1: 37,042$ & 60 \\
\hline Other fatty acid oxidation disorder & 2 & $1: 55,564$ & 40 \\
\hline \multicolumn{4}{|l|}{ 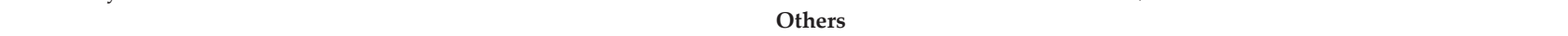 } \\
\hline Partial BD deficiency & 1 & 1:111,127 & 20 \\
\hline CFTR-Related Metabolic Syndrome (CRMS) & 5 & $1: 22,225$ & 100 \\
\hline Cystic Fibrosis & 5 & $1: 22,225$ & 100 \\
\hline Classical galactosemia & 1 & $1: 111,127$ & 20 \\
\hline Duarte galactosemia (D/G) & 2 & $1: 55,564$ & 40 \\
\hline Other disorders & 2 & $1: 55,564$ & 40 \\
\hline
\end{tabular}

${ }^{a}$ Based on an anticipated annual births of $2 \mathrm{M}$ and $100 \%$ coverage

Hemoglobin H-disease ${ }^{27,28}$ and anecdotal clinical experiences have revealed catastrophic outcomes for homozygous variants of these diseases (personal communication with Maria Beatriz P Gepte and Ernesto Yuzon, August 2012). In California, 109 cases of hemoglobinopathies were detected representing $54.8 \%$ of the 199 Filipino newborns with a confirmed diagnosis. It is anticipated that incorporation of hemoglobinopathies including Hemoglobin $\mathrm{H}$ Disease through multiplex screening tests will significantly increase hemoglobinopathy case detection in the Philippines, perhaps as high as 1860 cases annually, thereby significantly improving health outcomes through early diagnosis and treatment.

The Biochemical Genetics Unit of the Institute of Human Genetics ${ }^{29}$ contains a number of metabolic conditions in their listing of clinical cases including: PKU, MSUD, methylmalonic aciduria, citrullinemia, and many others. Unfortunately, these clinical patients usually present at a very late stage of disease often resulting in severe complications and death. Patients with PKU and MSUD are potentially more fortunate since screening tests for both of these disorders are currently included in the Philippine newborn screening panel. Thus, as parents of newborns become more aware of newborn screening and take advantage of the program, these patients have a much better chance for early detection and treatment leading to significantly improved health outcomes. Expanded screening for metabolic disorders will employ multiplex testing by MS/MS, such that a single sample punch for PKU will be simultaneously analyzed for more than 20 metabolic disorders including MSUD, Methyl Malonic Acidemia (MMA) and 20 others. In California, there were 6 cases of amino acid disorders (4 with PKU, 1 with variant hyperphenylalaninemia and 4 with MSUD); 7 cases of organic acid disorders ( 5 with MMA, 1 with $\beta$-ketothiolase deficiency (BKT) and 1 with Isobutyryl-CoA dehydrogenase deficiency (IBDHD); 10 cases of fatty acid oxidation disorders (FAOD), 2 with Medium chain acyl-CoA dehydrogenase (MCAD) deficiency, 3 with Short chain acylCoA dehydrogenase (SCAD) deficiency, 3 with Very Long chain acyl-CoA dehydrogenase (VLCAD) deficiency and 2 with other forms of fatty acid oxidation disorder); 1 case with partial biotinidase deficiency (BD), 1 case of classical galactosemia and 2 cases of Duarte galactosemia. This translates to an additional 580 disorders detectable each year in the Philippines including 140 cases of organic disorders, 
200 cases of fatty acid oxidation disorders and 240 other conditions.

The data on cystic fibrosis is interesting. There are no reported cases in the Philippines but there were 5 newborns diagnosed with cystic fibrosis in the CNSP. Upon review of the parental ethnicity of the 5 newborns, it was discovered that these patients were products of Filipino-Caucasian marriages. Given the increasing trend of mixed marriages, the addition of cystic fibrosis in the newborn screening panel may prove to be important for our population.

The benefit of the expanded screening program seems clear but this can be diminished due to the costs of implementation especially in a Low- to Middle-Income (LMIC) country setting like the Philippines. In the past decade, several cost-benefit analysis and cost-effectiveness studies were conducted to support expanded screening. In their study which evaluated the California screening program, Feuchtbaum and Cunningham ${ }^{30}$ reported that the benefits of using MS/MS outweighed the expenses incurred after their analysis revealed a benefit/cost ratio of US\$ 9.32. In 70 years, the costs spent on MS/MS newborn screening would be compensated for by the number of lives saved and morbidities avoided. ${ }^{31}$ Furthermore, another report stated that it was more cost-efficient to screen a group of diseases at once, rather than screen for single conditions separately, and that mass screening with MS/MS is even comparable to other screening programs such as that for breast and prostate cancer. ${ }^{32,33}$

\section{Conclusion}

Data from the CNSP showed that there are serious newborn disorders seen in Filipino babies that cannot be detected by the current newborn screening tests in the Philippines. Expanding the panel of disorders in the current Philippine newborn screening program to approximate that in the CNSP will save lives and reduce unnecessary negative health outcomes in Filipino newborns. This data was used for the development of an expanded newborn screening program for implementation in the Philippines.

The data on Filipino newborns taken from the CNSP was presented to the National Technical Working Group for Newborn Screening (NTWG-NBS) at the DOH. This data has prompted a review and subsequently a formal recommendation of expanded newborn screening program to the DOH Advisory Committee on Newborn Screening last 16 January 2012. A National Technical Working Group for Expanded Newborn Screening (NTWG-ENBS) was created under the National Center for Disease Prevention and Control (NCDPC) composed of personnel and staff from the Department of Health and representatives from different concerned institutions. The NTWG-ENBS is tasked to prepare the necessary guidelines for the implementation of expanded newborn screening in the country. The NTWG-
ENBS has been divided into several committees with specific responsibilities:

- Advocacy/Promotions - to prepare promotional programs prior to implementation.

- Regional Medical Centers - to develop guidelines for the regional medical centers that will be part of the follow-up system.

- Operations - to develop operational structure on fees, laboratory expansion and case follow-up.

On August 29, 2012, the DOH Advisory Committee approved the proposal for implementation. All NSCs are being reviewed for capability for expansion. To date, in preparation for implementation, two workshops featuring international experts have been held: 1) an orientation/workshop for metabolic disorders, which was attended by geneticists and neonatologists; and 2) an orientation/workshop for hemoglobinopathies, which was attended by geneticists and hematologists. Both workshops were attended by NSC heads, NSC laboratory and follow-up nurses. It is anticipated that seventeen multi-disciplinary regional medical centers will be organized as follow-up centers for the patients diagnosed by the newborn screening program. Expanded newborn screening is expected to be implemented in phases in 2013.

\section{Acknowledgments}

I wish to acknowledge and thank Dr. Bradford Therrell, U.S. National Newborn Screening and Genetics Resource Center, for facilitating communications with the CNSP and for reviewing and commenting on drafts of this manuscript. I also wish to thank Dr. Fred Lorey and Dr. Lisa Feuchtbaum, California Newborn Screening Program, for their cooperation and assistance in sharing their program data on Filipino newborns. I wish to acknowledge the support of the National Center for Disease Prevention and Control of the Department of Health and the staff of the Newborn Screening Reference Center, National Institutes of Health, University of the Philippines Manila.

\section{References}

1. American Academy of Pediatrics, Newborn Screening Task Force. Serving the family from birth to the medical home: Newborn screening: a blueprint for the future. Pediatrics. 2000; 106(suppl):389-427.

2. Guthrie R, Susi A. A simple phenylalanine method for detecting phenylketonuria in large populations of newborn infants. Pediatrics. 1963; 32:338-43.

3. Padilla CD, Therrell BL. Screening newborns in the Asia-Pacific Region. In: Kumar D, ed. Genomics and Health in the Developing World. Oxford: Oxford University Press, Inc. 2012:764-781.

4. Padilla CD, Basilio J, Oliveros Y. Newborn Screening: Research to Policy. Acta Med Philipp. 2009; 43(2):6-14.

5. Padilla CD. Towards universal newborn screening in developing countries: obstacles and the way forward. Ann Acad Med Singapore. 2008; 37(12Suppl):6-4.

6. Newborn Screening Reference Center (NSRC). Program Briefer. [Online]. [cited 2012 Dec]. Available from www.newbornscreening.ph. 
7. Schulze A, Lindner M, Kohlmuller D, Olgemoller K, Mayatepek E, Hoffman GF. Expanded newborn screening for inborn errors of metabolism by electrospray ionization-tandem mass spectrometry: results, outcome and implications. Pediatrics. 2003; 111(6 Pt 1):1399-406.

8. Benson JM, Therrell BL Jr. History and current status of newborn screening for hemoglobinopathies. Semin Perinatol. 2010; 34(2):134-44.

9. Lorey F, Cunningham G, Vichinsky EP, et al. Universal newborn screening for $\mathrm{Hb} \mathrm{H}$ disease in California. Genet Test. 2001; 5(2):93-100.

10. Hoppe CC. Newborn screening for hemoglobin disorders. Hemoglobin. 2011; 35(5-6):556-64.

11. Wilcken B, Wiley V, Hammond J, Carpenter K. Screening newborns for inborn errors of metabolism by tandem mass spectrometry. N Engl J Med. 2003; 348(23):2304-12

12. Bodamer OA, Hoffman GF, Lindner M. Expanded newborn screening in Europe 2007. J Inherit Metab Dis. 2007; 30(4):439-44

13. Therrell BL, Adams J. Newborn screening in North America. J Inherit Metab Dis. 2007; 30(4):447-65.

14. Huang HP, Chu KL, Chien YH, et al. Tandem mass neonatal screening in Taiwan-Report from one center. J Formos Med Assoc. 2006; 105(11):882-6.

15. Han LS, Ye J, Qiu WJ, Gao XL, Wang Y, Gu XF. Selective screening for inborn errors of metabolism on clinical patients using tandem mass spectrometry in China: a four year report. J Inherit Metab Dis. 2007; 30(4):507-14.

16. Lindmer M, Hoffman GF, Matern D. Newborn screening for disorders of fatty acid oxidation: experience and recommendations from an expert meeting. J Inherit Metab Dis. 2010; 33(5):521-6.

17. Feuchtbaum L, Carter J, Dowray S, Currier RJ, Lorey F. Birth prevalence of disorders detectable through newborn screening by race/ethnicity. Genet Med. 2012; 14(11):937-45.

18. Watson MS. Current status of newborn screening: decision-making about the conditions to include in screening programs. Ment Retard Dev Disabil Res Rev. 2006; 12(4):230-5.

19. Loeber JG. Neonatal screening in Europe: the situation in 2004. J Inherit Metab Dis. 2007; 30(4):430-8.

20. Borrajo GJ. Newborn screening in Latin America at the beginning of the 21st century. J Inherit Metab Dis. 2007; 30(4):466-81.
21. Saadallah AA, Rashed MS. Newborn screening: experiences in the Middle East and North Africa. J Inherit Metab Dis. 2007; 30(4):482-9.

22. Wilcken B. Recent advances in newborn screening. J Inherit Metab Dis 2007; 30(2):129-33.

23. Yamaguchi S. Newborn Screening in Japan: restructuring for the new era. Ann Acad Med Singapore. 2008; 37(12 Suppl ):13-5.

24. Gu X, Wang Z, Ye J, Han L, Qiu W. Newborn Screening in China: phenylketonuria, congenital hypothyroidism and expanded screening. Ann Acad Med Singapore. 2008; 37(12 Suppl):107-4.

25. Yoon HR, Lee KR, Kang S, et al. Screening of newborns and high-risk group of children for inborn metabolic disorders using tandem mass spectrometry in South Korea: a three-year report. Clin Chim Acta. 2005; 354(1-2):167-80

26. Han M, Jun SH, Song SH, Park KU, Kim JQ, Song J. Use of tandem mass spectrometry for newborn screening of 6 lysosomal storage disorders in a Korean population. Korean J Lab Med. 2011; 31(4):250-6.

27. Vichinsky E. Advances in the treatment of alpha-thalassemia. Blood Rev. 2012; 26 Suppl 1:S31-4

28. Lal A, Goldrich ML, Haines DA, Azimi M, Singer ST, Vichinsky EP. Heterogeneity of hemoglobin $\mathrm{H}$ disease in childhood. N Engl J Med. 2011; 364(8):710-8.

29. Padilla CD, de la Paz EM. Genetic Services and testing in the Philippines. J Community Genet. 2012. DOI 10.1007/s12687-012-0102-1.

30. Feuchtbaum L, Cunningham G. Economic evaluation of tandem mass spectrometry screening in California. Pediatrics. 2006; 117(5 Pt 2):S280-6.

31. Venditti LN, Venditti CP, Berry GT, et al. Newborn screening by tandem mass spectrometry for medium-chain Acyl-CoA dehydrogenase deficiency: a cost-effectiveness analysis. Pediatrics. 2003; 112(5):1005-15.

32. Cipriano LE, Rupar CA, Zaric GS. The cost-effectiveness of expanding newborn screening for up to 21 inherited metabolic disorders using tandem mass spectrometry: results from a decision-analytic model. Value Health. 2007; 10(2):83-97.

33. Schoen EJ, Baker JC, Colby CJ, To TT. Cost-benefit analysis of universal tandem mass spectrometry for newborn screening. Pediatrics. 2002; 110(4):781-6. 


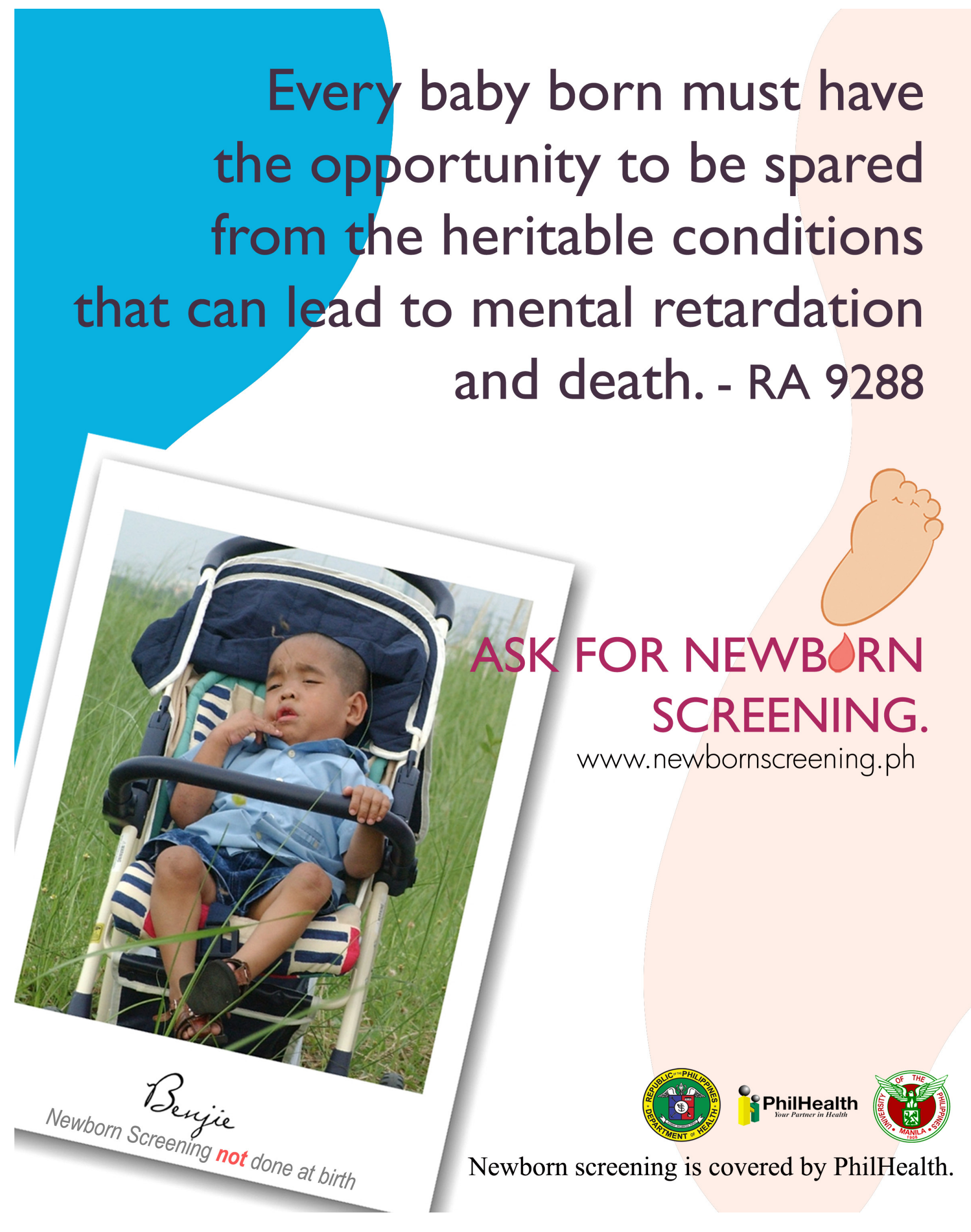

